\title{
Hydrate nucleation and growth on water droplets acoustically- levitated in high-pressure natural gas
}

Received 00th January 20xx, Accepted 00th Jaary 20xx

DOI: $10.1039 / x 0 x \times 00000 x$

\author{
Kwanghee Jeong, ${ }^{a}$ Peter J. Metaxas, ${ }^{\text {a Joel Chan, }}{ }^{\text {a }}$ Temiloluwa O. Kuteyi, ${ }^{\text {a }}$ Zachary M. Aman, ${ }^{\text {a }}$ Paul L. \\ Stanwix, ${ }^{a}$ Michael L. Johns, ${ }^{a}$ and Eric F. May*a
}

Hydrate formation was studied using water droplets acoustically levitated in high-pressure natural gas. Despite the absence of solid interfaces, the droplets' area-normalised nucleation rate was about four times faster than in steel autoclave measurements with interfacial areas roughly 200 times larger. Multiple stages of stochastic, template-free hydrate growth were observed.

Clathrate hydrates of natural gas are solid, ice-like compounds in which guest gas molecules (e.g. methane, ethane, carbon dioxide) are enclathrated within cage-like structures consisting of hydrogen bonded water molecules (1). Thermodynamically stable at temperatures and pressures encountered in natural gas production or at oceanic or permafrost margins (2), hydrates are highly relevant to applications in conventional energy production from oil and gas reserves (flow assurance (3)), unconventional energy production from geological deposits of methane-hydrate $(4,5)$ and to onshore processes related to gas storage (6) and water desalination (7). The kinetics of hydrate formation are critical to many of these applications, including optimising gas storage schemes, reducing the cost of hydrate management strategies for subsea gas production (8) or avoiding blockages in the cryogenic heat exchangers used to make liquefied natural gas $(L N G)(9,10)$.

A major challenge in characterising hydrate formation experimentally is that it begins with a stochastic (11) nucleation process (12). This means that large numbers of independent hydrate formation events must be measured to map out formation probability distributions as a function of system conditions. Furthermore, relative to other crystallisation processes, the nucleation rates of natural gas hydrates are slow, because of their multi-component nature and the low solubility of the guest molecules in the aqueous liquid. Thus, acquiring a sufficient number of representative formation data to adequately characterise this stochastic process can be difficult using traditional high-pressure apparatus due to their slow temperature ramping rates $(13,14)$ or the inability to apply shear (15).

\footnotetext{
a. Fluid Science \& Resources, School of Engineering, University of Western Australia,
} 35 Stirling Highway, Perth, WA 6009, Australia.E-mail: eric.may@uwa.edu.a
Recently, May and co-workers $(16,17)$ reported robust measurements of gas hydrate formation probability distributions using an improved technique based on a highpressure stirred automated lag time apparatus (HPS-ALTA), and compared their results with other experimental studies and the predictions of classical nucleation theory (CNT). (11) They found that careful nucleation rate measurements reported in the literature were broadly consistent, particularly those determined in well-mixed systems where the agreement was within a factor of three. The observations were also consistent with the CNT predictions of nucleation work (energy barrier). However, the kinetic parameter predicted by CNT-based theory for hydrate nucleation was around 20 orders of magnitude larger than observed.

For a fixed temperature, $T$, and pressure, $p$, within the hydrate stability region, CNT states that the probability nucleation will have occurred after a given induction time, $P(t)$, is given by an exponential distribution (18)

$$
P(t)=1-\exp (-J t)(1)
$$

where $J$ is a nucleation rate specific to the system sub-cooling $\Delta T=T_{\text {eqbm }}-T$, and where $T_{\text {eqbm }}$ is the hydrate equilibrium temperature at $p$. This distribution is indeed observed if sufficient numbers of formation events are measured, particularly if the experimental system is well-mixed and devoid of mass transfer limitations. (16,19-21) The nucleation rate scales with system size, and in principle should be proportional to either volume, $V$, in the case of homogenous nucleation (difficult to achieve in practice (22)), or to the area, $A$, of the interface upon which nucleation is occurring. However, a key limitation identified by Metaxas et al. (16) upon analysing multiple hydrate formation experiments was the inability to identify the specific interface at which the nucleation was occurring. Similar absolute hydrate nucleation rates (around $0.001 \mathrm{~s}^{-1}$ at $\Delta T=10 \mathrm{~K}$ ) were observed using various apparatus despite them having very different gas-water or water-steel interfacial areas, which are the two interfaces where hydrate nucleation is likely to occur in a well-stirred system. (11) 
a
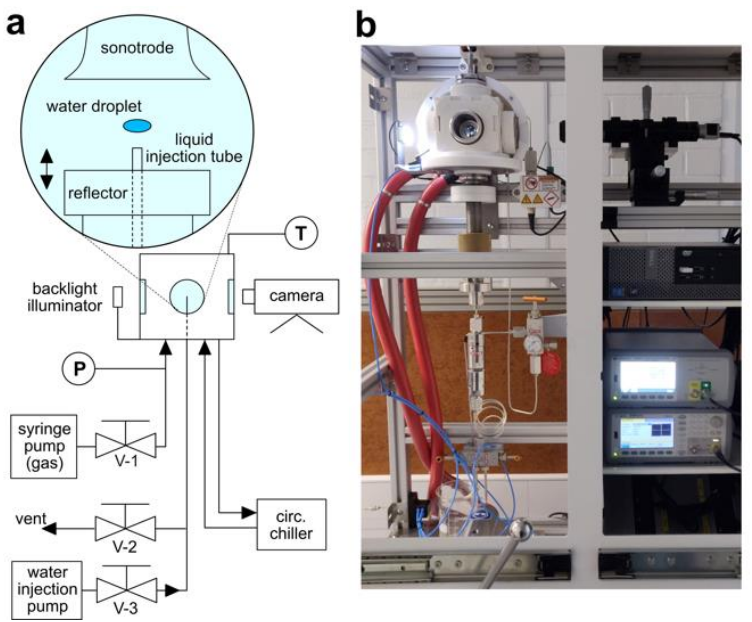

Figure 1. System schematic (a) and system photo (b) showing the levitation cell, high pressure injection lines, electronics and camera. $\mathrm{P}$ and $\mathrm{T}$ indicate the pressure and temperature measurement locations.

The presence of nucleation sites on the interface (assumed) responsible for nucleation, is often posited as an explanation for the area-normalised nucleation rates $(J / A)$ observed. These sites are locations on the interface at which the required nucleation energy barrier is much lower than elsewhere, and are often thought to correspond to imperfections on the solid surface being used to contain the fluids. (11) However, such explanations imply that measurements of hydrate formation will be apparatus-specific and virtually impossible to generalise given the inability to characterise these sites or even be certain about the interface where nucleation is occurring. In this work, we aimed to address both issues by measuring hydrate formation on water droplets acoustically levitated in highpressure natural gas. In such an experiment the interface where nucleation occurs is the droplet surface, which has a quantifiable area. Hence the observed $(J / A)$ cannot be attributed to any imperfections on a solid surface.

Previous studies aiming at such measurements in highpressure systems have looked at hydrate formation in "quasifree" water droplets, which are either resting on hydrophobic solid surfaces $(23,24)$ or suspended within (hydrophobic) liquids (25-27). Somewhat analogous measurements have also been made on suspended gas bubbles in water (28). Here, we present the first measurement of hydrate formation probability in a water droplet fully suspended within a high pressure gas phase. This is made possible using a high pressure acoustic levitator which, unlike ambient-pressure systems (29), enables the study of structure I (sI) or structure II (sII) hydrates that form under

a

$$
\text { b }
$$

C

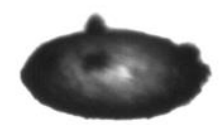

$\mathrm{t}=5 \mathrm{~s}$

high pressure from either methane or more complex naturalgas-like mixtures.

The high-pressure acoustic levitator (L800; BOROSA Acoustic Levitation $\mathrm{GmbH}$ ) is shown in Figure 1. The levitator's sonotrode and reflector were housed within a thermally insulated, temperature controlled, high pressure measurement cell. The sonotrode was driven by an amplified waveform generator (33500B/33502A, Keysight) at a frequency around $34.5 \mathrm{kHz}$. Temperature control was achieved using a circulating chiller, which delivered a heat-transfer fluid to a cooling jacket around the cell. The cell temperature was measured using a Ktype thermocouple inserted into the cell wall. The size of any temperature offset between the levitated droplet and the cell wall was tested by conducting ice melting and hydrate dissociation measurements. These showed that any offset was between ( 0.2 and 0.7$) \mathrm{K}$ for the temperature range ( 0 to 21$)^{\circ} \mathrm{C}$.

Pressure was measured using a transducer $(0-100 \mathrm{MPa}$, $0.2 \%$ uncertainty, CPT2500, WIKA) located near the cell. Windows in the side of the cell allowed for imaging of backilluminated acoustically levitated droplets. Pressure, temperature, and droplet images were recorded every 5-10 seconds during the measurements. Droplet volumes and surface areas were calculated from the images under the assumption of radial symmetry using proprietary analysis software (BOROSA Acoustic Levitation $\mathrm{GmbH}$ ). A sll forming synthetic natural gas mixture was used for all experiments (mole-fraction composition of $0.78 \mathrm{CH}_{4}+0.12 \mathrm{C}_{2} \mathrm{H}_{6}+0.06 \mathrm{C}_{3} \mathrm{H}_{8}$ $+0.01 \mathrm{i}-\mathrm{C}_{4} \mathrm{H}_{10}+0.03 \mathrm{CO}_{2}$ ). The small amount of $\mathrm{CO}_{2}$ present in the gas meant that the concentration of carbonic acid in the droplet was negligible.

To carry out a formation measurement, the water pump was first filled with deionised water and the valve (V-3) closed (Figure 1a). The cell wall temperature was then allowed to stabilise at an average value of $(7.1 \pm 0.3){ }^{\circ} \mathrm{C}$, where the error bound here and below corresponds to a standard deviation across the 29 independent measurements. Cell pressurisation then was carried out via V-1. The cell was first purged three times with $1.3 \mathrm{MPa}$ of the gas mixture before being pressurised to approximately $(6.5 \pm 0.2) \mathrm{MPa}$. Once the pressure and temperature were stable, V-3 was opened and water was gradually pumped into the cell, enabling the introduction of a single droplet into the acoustic field (Figure 2a). Formation onset was identified based on the appearance of visible solids. For example, in the experiment from which the images in Figure 2 were taken, the first sign of solids formation is in the snapshot shown in Figure $2 b$ where a partial shell has formed on the water droplet. The induction time was defined experimentally

d

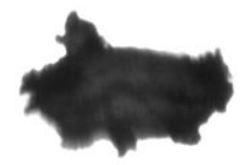

$t=28 \operatorname{mins} 30 \mathrm{~s}$ e

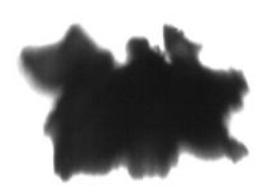

$\mathrm{t}=1 \mathrm{hr} 45$ mins f

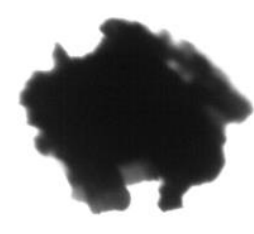

$\mathrm{t}=1 \mathrm{hr} 54$ mins

Figure 2. Back illuminated images of (a) the water droplet prior to detectable hydrate formation onset, (b) the droplet with a partial hydrate shell (formation onset for this particular measurement), and (c-f) snapshots showing the evolution of the growing hydrate particle. The scale bar shown in panel (a) is $1 \mathrm{~mm}$ long. 
as the time between commencing water injection (and thus first exposing the water to the high pressure gas phase) and observing the first solids on the droplet interface.

a
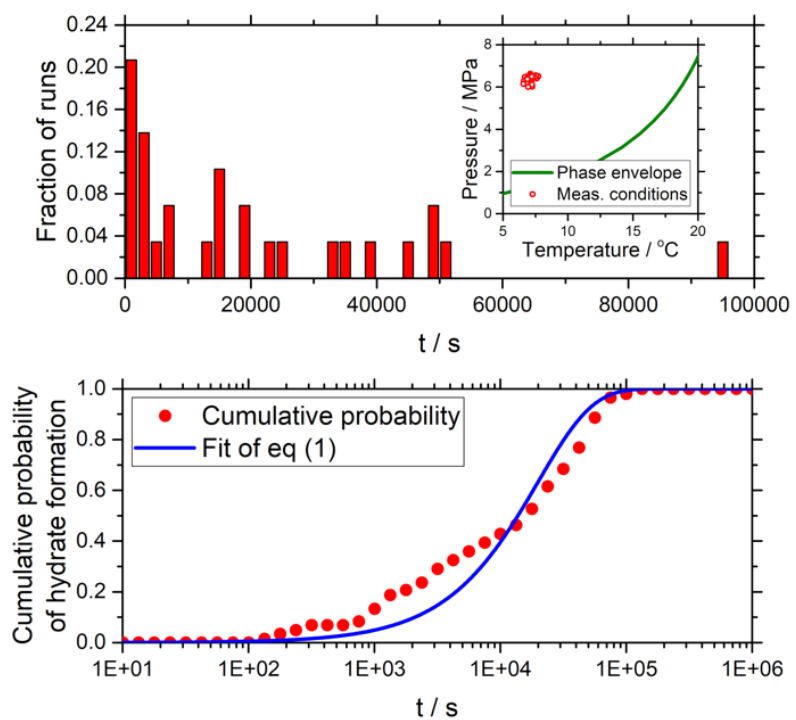

Figure 3. (a) Histogram showing the distribution of measured induction times. The inset shows the pressure and temperature conditions for each of the 29 levitation experiments relative to the hydrate equilibrium curve. (b) Cumulative gas hydrate formation probability distribution versus time with fit of eq (1)

The induction time measurements were obtained at a subcooling of $(12.0 \pm 0.3) \mathrm{K}$ and a droplet volume of $(3.5 \pm$ 1.4) $\mathrm{mm}^{3}$. The inset of Figure $3 a$ shows the hydrate phase envelope together with the measurement conditions for the 29 experiments. The equilibrium curve shown was calculated using the Cubic Plus Association (CPA) model implemented in the software Multiflash 6.2 (KBC). The hydrate equilibrium temperature for this gas was measured to be $20.8^{\circ} \mathrm{C}$ at $7.2 \mathrm{MPa}$ in a separate experiment following the method of Akhfash et al. (30) using a sapphire autoclave; this is within $0.9 \mathrm{~K}$ of the predicted value and confirms that the hydrate formed was sll.

The measured induction times exhibit a large spread, ranging from $130 \mathrm{~s}$ to $95,420 \mathrm{~s}$. The mean and standard deviation of the distribution converged to their final values after 25 points, confirming that a sufficient number of formation events were acquired. The similarity of the mean $(19,669 \mathrm{~s})$ and standard deviation $(22,204 \mathrm{~s})$ is indicative of exponentially distributed induction times, consistent with eq 1 . The corresponding histogram is shown in Figure 3a, where the measured induction times have been grouped using 2,000 s wide bins. Figure $3 \mathrm{~b}$ shows the cumulative probability of hydrate formation plotted as a function of induction time. The latter has been determined by trapezoidal integration (15) of a second histogram (not shown) constructed using bins that increased in width logarithmically. This avoids the sharp jumps in cumulative probability that result from the sparselydistributed (16) high-induction-time events present in the linearly binned data shown in Figure 3a.

We fit the cumulative probability data in Figure $3 \mathrm{~b}$ with eq. (1), finding a reasonable agreement with theory notwithstanding the $\pm 0.3 \mathrm{~K}$ variation in subcooling. The absolute nucleation rate determined by the fit was $J=(5.0 \pm$ 0.3) $\times 10^{-5} \mathrm{~s}^{-1}$, where the error bound denotes the statistical uncertainty of the fit parameter. The average interfacial area of the slightly elliptical droplets was determined to be $A=(11.3 \pm 3.1) \mathrm{mm}^{2}$, giving an area-normalised nucleation rate of $(J / A)=(4.4 \pm 1.2) \mathrm{m}^{-2} \mathrm{~s}^{-1}$ at $\Delta T=12.0 \mathrm{~K}$.

By normalising to the $20 \mathrm{~cm}^{2}$ wetted steel interfacial area of the HPS-ALTA, Metaxas et al. (16) reported a $(J / A)=(0.46 \pm 0.01) \mathrm{m}^{-2} \mathrm{~s}^{-1}$ at $\Delta T=9.8 \mathrm{~K}$; at the subcooling applied in this work, their data suggest values of $(J / A)$ of less than $1 \mathrm{~m}^{-2} \mathrm{~s}^{-1}$. Thus, the area-normalised nucleation rate observed in the HPS-ALTA with $20 \mathrm{~cm}^{2}$ of wetted steel was more than 4.4 times lower than the area-normalised nucleation rate measured for an $11 \mathrm{~mm}^{2}$ acoustically-levitated droplet.

While the precision of the current measurements precludes more stringent comparisons with HPS-ALTA or autoclave data, the key result obtained here is that the area-normalised nucleation rates for two qualitatively different experiments was within a factor of 5 even though the interfacial areas differed by a factor of nearly 200. In particular, the absence of any influence by a solid container on the formation of hydrates on the acoustically-levitated droplets implies that any nucleation sites responsible for the $(J / A)$ values observed should not be attributed to surface defects. Rather the sites driving the observed nucleation rates should be common to both systems, with one likely candidate being unresolved bubbles entrained near the gas water interface by the shear applied. The shear field relevant in this work is due to the oscillating acoustic field, and the hypothesis of entrained microbubbles as the primary nucleation site is consistent with the observation in previous a

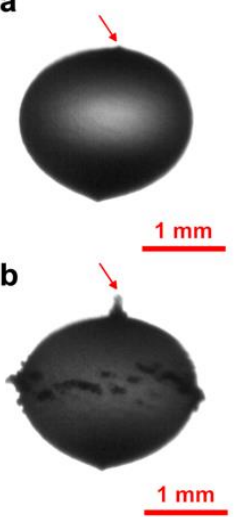

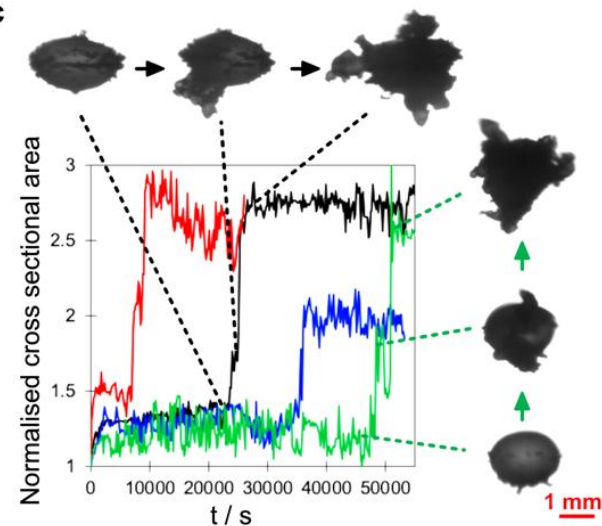

Figure 4. Stochastic hydrate shell growth. The pointed part of the hydrate shell in (a) is the site of the first major outward growth from the particle in (b). The evolution of the particle cross-sectional area versus time since formation onset for four hydrate particles is shown in (c). This enables the transition to a large-area, highly roughened morphology to be identified. Corresponding snapshots for the particles are shown as insets.

studies of ice nucleation using ambient-pressure acoustic levitation, which found that the ultrasonic sound pressure level influenced the nucleation rate $(31),(32,33)$. In an industrial system, the entrainment of bubbles is associated with the high shear caused by turbulent multi-phase flow.

Following formation onset, complete shell growth typically took 10-20 s. This shell growth rate is of the same order of magnitude as that seen previously for $\mathrm{mm}$-scale droplets at a 
sub-cooling of approximately $15 \mathrm{~K}(34)$. Figure $2 \mathrm{c}-\mathrm{f}$, shows that following shell formation, the particle started to expand in a non-uniform manner and thus roughened. This non-uniform, outward growth, which initiated via discrete localised growth events, was presumably driven by water exiting the droplet via defects (or potentially pores (34)) in the shell (24), thus providing a source of free water to the outside gas phase for continued hydrate growth. Evidence for defects acting as sites for growth onset can be seen in Figure 4a-b, where the initial outward growth of the shell occurred at a pointed part of the otherwise smoothly-curved hydrate shell. Cross-sectional areas of the droplet converting to a particle were measured as a function of time after formation onset using algorithms (35) implemented in the ImageJ software package, enabling a clear identification of the time, $t_{\text {rough, }}$ at which the particle transitioned to a highly roughened state with a significantly increased surface area. Four examples of this transition are shown in Figure 4c, which highlights the stochastic nature of the transition to a large area particle; $t_{\text {rough }}$ varies by an order of magnitude across the four experiments shown, which can likely be linked to variations in the shell structure in the different particles. This result could help explain the stochastic hydrate growth rate distributions recently observed in HPS-ALTA experiments. (16)

In summary, we have presented the first measurements of hydrate formation in a water droplet that has been acoustically levitated in a high pressure natural gas mixture. The induction time for hydrate formation onset and transitions in the hydrate particle morphology were both found to be stochastic, the distribution of the former being consistent with predictions from Classical Nucleation Theory (eq 1) and the latter presumably being linked to variations in hydrate shell integrity across different particles. The area-normalised hydrate nucleation rate was calculated from the induction time distribution and found to be within a factor of 5 of conventional rates measured in the presence of a steel-water interface. Based on this similarity, we hypothesise that microbubbles entrained by shear are a more important source of nucleation sites than defects on steel surfaces. This has implications on how hydrate nucleation rates in large-industrial systems might be estimated, based on the degree of shear present together with the gas-water interfacial area rather than the available water-steel interface.

This research was funded by the Australian Government through the Australian Research Council (LE170100174, FT180100572 and IC150100019). The authors thank D. Borosa for advice regarding the acoustic levitator.

\section{Conflicts of interest}

There are no conflicts to declare.

\section{Notes and references}

1. Sloan ED. Nature. 2003;426(6964):353-9.

2. May EF, Marsh KN, Goodwin ARH. Future Energy (Second Edition): Improved, Sustainable and Clean Options for our Planet2014. p. 75-93.
3. Sloan ED, Koh C, Sum AK, Ballard AL, Creek J, Eaton M, et al. Natural Gas Hydrates in Flow Assurance. 2011:1-200.

4. Milkov AV. Earth-Science Reviews. 2004;66(3):183-97.

5. Stanwix PL, Rathnayake NM, de Obanos FPP, Johns ML, Aman ZM, May EF. Energy \& Environmental Science. 2018;11(7):1828-40.

6. Linga P, Kumar R, Lee JD, Ripmeester J, Englezos P. International Journal of Greenhouse Gas Control. 2010;4(4):630-7.

7. Jeong K, Choo YS, Hong HJ, Yoon YS, Song MH. Review of Scientific Instruments. 2015;86(3):035102.

8. Kinnari K, Hundseid J, Li X, Askvik KM. Journal of Chemical \& Engineering Data. 2015;60(2):437-46.

9. Baker CJ, Oakley JH, Rowland D, Hughes TJ, Aman ZM, May EF. Energy \& Fuels. 2018;32(1):255-67.

10. Baker C, Siahvashi A, Oakley J, Hughes T, Rowland D, Huang S, et al. The Journal of Chemical Thermodynamics. 2019;137:22-33.

11. Kashchiev D, Firoozabadi A. J Cryst Growth. 2002;243(3-4):47689.

12. Karthika S, Radhakrishnan TK, Kalaichelvi P. Cryst Growth Des. 2016;16(11):6663-81.

13. Abay HK, Svartaas TM. Energy \& Fuels. 2010;24:752-7.

14. Lone A, Kelland MA. Energy \& Fuels. 2013;27(5):2536-47.

15. May EF, Wu R, Kelland MA, Aman ZM, Kozielski KA, Hartley PG, et al. Chemical Engineering Science. 2014;107:1-12.

16. Metaxas PJ, Lim VWS, Booth C, Zhen J, Stanwix PL, Johns ML, et al. Fuel. 2019;252:448-57.

17. May EF, Lim VW, Metaxas PJ, Du J, Stanwix PL, Rowland D, et al. Langmuir. 2018;34:3186.

18. Kashchiev D, Firoozabadi A. J Cryst Growth. 2003;250(3-4):499515.

19. Ke W, Svartaas TM, Kvaloy JT, Kosberg BR. Energy \& Fuels. 2016;30(9):7646-55

20. Svartaas TM, Ke W, Tantciura S, Bratland AU. Energy \& Fuels. 2015;29(12):8195-207.

21. Maeda N, Shen X-d. Fuel. 2019;253:1597-604.

22. Knott BC, Molinero V, Doherty MF, Peters B. J Am Chem Soc. 2012;134(48):19544-7.

23. Tanaka R, Sakemoto R, Ohmura R. Cryst Growth Des. 2009;9(5):2529-36.

24. Servio P, Englezos P. Aiche J. 2003;49(1):269-76.

25. Maeda N. Review of Scientific Instruments. 2014;85(6):065115.

26. Maeda N. Aiche J. 2015;61(8):2611-7.

27. Maeda N. Chemical Engineering Science. 2016;155:1-9.

28. Chen L, Levine JS, Gilmer MW, Sloan ED, Koh CA, Sum AK. Journal of Chemical \& Engineering Data. 2014;59(4):1045-51.

29. Sarfraz A, Schlegel MC, Wright J, Emmerling F. Chemical Communications. 2011;47(33):9369-71.

30. Akhfash M, Arjmandi M, Aman ZM, Boxall JA, May EF. J Chem Eng Data. 2017;62(9):2578-83.

31. Lü YJ, Xie WJ, Wei B. Applied Physics Letters. 2005;87(18):184107.

32. Chow R, Mettin R, Lindinger B, Kurz T, Lauterborn W, editors. IEEE Symposium on Ultrasonics, 2003; 2003 5-8 Oct. 2003.

33. Inada T, Zhang X, Yabe A, Kozawa Y. Int J Heat Mass Tran. 2001;44(23):4523-31.

34. Lee JD, Susilo R, Englezos P. Chemical Engineering Science. 2005;60(15):4203-12.

35. Schneider CA, Rasband WS, Eliceiri KW. Nature Methods. 2012;9:671. 